\title{
I recognize your face, but I can't remember your name: A question of expertise?
}

\author{
Rasha Abdel Rahman \\ Max Planck Institute for Psycholinguistics, Nijmegen, The Netherlands \\ Werner Sommer \\ Humboldt-Universität zu Berlin, Berlin, Germany \\ Ella Olada \\ University of Krasnojarsk, Krasnojarsk, Russia
}

\begin{abstract}
In four experiments on the identification of familiar faces we reassessed a robust performance pattern-namely, the temporal advantage for retrieving biographical facts as compared to recalling proper names, which has been interpreted as reflecting a serial ordering of the access to semantic and name information. Evidence for recent parallel accounts had been provided by Scanlan and Johnston (1997) who reported an advantage for name retrieval in children. Here we replicated the findings of Scanlan and Johnston but also showed that the naming advantage disappears, and performance is very similar to that of adults when stimuli and tasks are used that are familiar to children. Conversely, we also demonstrated an advantage for name retrieval in adults when highly unfamiliar semantic facts were associated with the faces. Together these findings suggest that there is no fundamental difference in the cognitive architectures of children and adults. The experiments indicate that the relative speed of naming and semantic fact retrieval depends on the expertise with the semantic facts to be retrieved. Implications for models of face identification and naming are discussed.
\end{abstract}

The ability to recognize and identify familiar faces as belonging to, for example, a politician named "Tony Blair" is an essential skill for everyday life. Research on face-naming performance suggests that, in contrast to biographical information such as occupation or nationality, proper name retrieval is relatively slow and susceptible to memory blocks in both healthy individuals (Brédart, 1993; Hanley \& Cowell, 1988; Young, Hay, \& Ellis, 1985) and braindamaged patients (Fery, Vincent, \& Brédart, 1995; Hanley, 1995; Luchelli \& De Renzi, 1992). Thus, deficient naming in the context of intact access to biographical information has been

Correspondence should be addressed to Rasha Abdel Rahman, Max Planck Institute for Psycholinguistics, PO Box 310, 6500 AH Nijmegen, The Netherlands. Email: rasha.abdel-rahman@mpi.nl

This research was supported by a PhD fellowship (Graduiertenkolleg 423) of the Deutsche Forschungsgemeinschaft to Rasha Abdel Rahman and of the German academic exchange service to Ella Olada. We appreciate the help of Friederike Engst in data collection.

(C) 2004 The Experimental Psychology Society 
repeatedly demonstrated (e.g., Semenza \& Zettin, 1988, 1989). In contrast, the reverse dissociation - that is, the ability to name a face in the absence of biographical knowledge about the person, has never been reported for normal subjects and is controversially discussed in the neuropsychological literature (cf. Brennen, David, Fluchaire, \& Pellat, 1996; Hodges \& Greene, 1998). Furthermore, binary decisions about biographical facts (e.g., nationality or occupation) are usually faster than classifications of names and overt naming (Johnston \& Bruce, 1990; Young, Ellis, \& Flude, 1988; Young, McWeeny, Ellis, \& Hay, 1986).

The seminal Bruce and Young (1986) model of face recognition and identification accounts for these observations by suggesting that access to biographical—or semanticfacts and proper names is ordered in series. Crucially, the naming of familiar faces is assumed to be contingent on the retrieval of identity-specific semantic information associated with the person. More recently, several authors have suggested that semantic information and names can be retrieved in parallel (Brédart, Valentine, Calder, \& Gassi, 1995; Burton \& Bruce, 1992, 1993; Burton, Bruce, \& Hancock, 1999), by adopting central assumptions of interactive activation and competition (IAC) models (McClelland \& Rumelhart, 1981). A common feature of these IAC models is that access to both identity-specific semantic information and people's names is mediated by so-called person identity nodes (PINs). These nodes do not represent any specific information but merely direct the activation flow into units representing biographical facts and names. IAC models explain the disadvantage for naming as compared to semantic fact retrieval with structural properties of the networks. Typically, names are uniquely associated with just one person whereas many biographical facts, such as nationality or profession, are shared by several persons. Therefore, the units representing name information have fewer connections than units representing semantic information (SIUs). It is this uniqueness of names that makes them more difficult to retrieve. Thus, the robust finding of slower name retrieval can be accommodated by both serial and parallel models.

Unfortunately, empirical evidence is scant that would allow distinguishing between serial and parallel models. Schweinberger, Burton, and Kelly (2001) have shown semantic priming effects on classifications of semantic categories such as nationality but not, as should have been expected from a serial point of view, on classifications of name phonology. Recently, Abdel Rahman, Sommer, and Schweinberger (2002) presented psychophysiological data in support of parallel access to names and biographical facts. In so-called two-choice go/no-go tasks binary decisions about both semantic facts and names of politicians were required. Crucially, the duration of the presumably mediating process, the retrieval of semantic information, was manipulated. For example, in one experiment the response hand was determined by a semantic classification that was either relatively fast (foreign vs. domestic) or relatively slow (member of governing vs. opposition party). In contrast, the decision about executing or withholding the response (go vs. no-go) was based on a binary name classification; for example, the selected response was to be executed if the first vowel of the person's name was an $a$ but was to be withheld if it was an $e$. Along with the participant's behaviour we extracted the so-called lateralized readiness potential (LRP) from the electroencephalogram, which is considered to index response activation at the level of the motor cortex. In the two-choice go/no-go task the LRP reveals temporal information about the relative processing duration of semantic and phonological information retrieval. At variance with a serial processing account the LRP in both types of trial indicated that the beginning of name retrieval does not depend on the 
duration of semantic processing. This is most clearly demonstrated in the experiment described here as an example of where the interval between LRP onset - indicating the termination of semantic retrieval - and the response execution depended on the type of semantic classification. The parallel architecture of modern IAC models (e.g., Brédart et al., 1995; Burton et al., 1999) can easily accommodate this finding.

Scanlan and Johnston (1997) presented another interesting piece of evidence against serial models. In striking contrast to the advantage for retrieving semantic information over proper names that is commonly observed in adults, children under 13 showed an advantage for name retrieval. Scanlan and Johnston presented photographs of celebrities together with a printed word, designating a name, an occupation, or a nationality. Participants were instructed to classify the face-name, face-occupation, or face-nationality pairings as correct or incorrect. Whereas adults produced the expected pattern of shorter reaction times for semantic than for name classifications, children were faster in matching faces to names than in matching faces to occupations and nationalities. This finding is difficult to explain within the framework of a discrete serial model because it would require the implausible assumption of a reordering of processing stages during adolescence. In contrast, it is easy to accommodate Scanlan and Johnston's finding with IAC models, in which both types of information are retrieved in parallel. One would have to assume only that in children semantic retrieval is more timeconsuming than is name retrieval; this, in turn, might relate to immature SIUs or connections between PINs or SIUs.

In the light of the controversial theoretical conceptions about name and biographical fact retrieval it would be of greatest interest to replicate the findings of Scanlan and Johnston (1997) and to assess whether they also hold for stimulus material and tasks that are more familiar to children. In the semantic tasks of Scanlan and Johnston's Experiment 2, the children appeared to have exceptional difficulties, producing error rates of up to $34 \%$. In Experiment 3 this problem was addressed by familiarizing both age groups with the presented stimuli; the children's naming advantage was replicated but their error rates were still up to $19 \%$ as compared to a mere $1 \%$ in the adult participants. Without questioning the validity of these findings, the high error rates clearly illustrate the difficulties that children experience with these tasks. A second concern is related to Scanlan and Johnston's semantic classification tasks. Categories like occupation or nationality might be both unfamiliar and artificial to children, thus causing the observed effects by increasing reaction time specifically in these conditions.

Therefore, the present study assessed whether the name retrieval advantage in children reported by Scanlan and Johnston (1997) also holds for more familiar stimulus and task conditions. A developmental change in cognitive architecture might occur for familiar face identification and naming, and, therefore, children may retrieve semantic and name information in a substantially different way from that of adults. In this case the results of Scanlan and Johnston should also hold for familiar stimuli and tasks. Alternatively, and as explained above, the results obtained by Scanlan and Johnston might be due to the unfamiliarity of the children with the stimuli and tasks. For example, it has been argued that for object naming the use of age-appropriate stimuli is essential to determine whether differences between children and adults can be attributed to differential properties of the cognitive architectures (Cycowicz, Friedman, Rothstein, \& Snodgrass, 1997). Thus, Graf (1990) observed comparable priming effects in children and adults only when the stimulus material was normalized for both age 
groups. If this reasoning holds, adults and children should perform similarly when appropriate stimuli and semantic categorization tasks are employed.

Because it is difficult to find a set of real persons that are highly familiar to all children participating in the study, Experiments 1 and 2 of the present study employed faces of cartoon characters. Earlier research on this type of stimulus with adults suggests that, despite greater visual heterogeneity, cartoon characters share important characteristics with faces regarding the time course of semantic processing and name retrieval. For example, when measuring the time required to name cartoon characters, as compared to classifying them according to a semantic category, Johnston (1994) observed the common advantage of semantic over name retrieval. A major shared characteristic of celebrities and cartoon characters is that both are processed at an individual, identity-specific level. In contrast, common objects are processed and named at an identity-unspecific, so-called basic level. Valentine, Brennen, and Brédart (1996) and Valentine, Hollis, and Moore (1998) emphasized that the naming of familiar faces and common objects differs in that identity-specific nodes (so-called "token markers") mediate parallel retrieval of semantic and name information for familiar faces whereas no such token markers exist for common objects. Therefore, familiar cartoon characters like Donald Duck should be identified (as living in Duck Town, having a rich uncle and a girl friend named Daisy, etc.) and named at an identity-specific level, in a similar way as are portraits of familiar persons.

In Experiment 1 adult performance in naming and semantic classification of cartoon characters was examined. If these characters are processed in a similar way as faces, serial as well as parallel models predict faster classification latencies for semantic attributes than for names. The crucial question addressed in Experiment 2 was whether the same effect holds true also for children or whether their previously reported naming advantage can be replicated.

\section{EXPERIMENT 1}

\section{Method}

\section{Participants}

Eight females and four males, aged 20 to 45 years $(M=28)$ were paid for their participation in the experiment or received partial fulfilment of a curriculum requirement. All participants were native German speakers and reported normal or corrected-to-normal vision.

\section{Stimuli}

Eight well-known cartoon characters were selected as stimuli-namely Mickey Mouse, Goofy, Donald Duck, Huey (Tick), Asterix, Obelix, Hein Blöd, and Käpt'n Blaubär (the latter two are from a popular German TV series). For each character, three drawings of the face from different viewpoints were scanned from comic strips and books and edited to 8-bit colour images with a homogeneous blue background. The images were scaled to $3.5 \times 3.5 \mathrm{~cm}$.

The cartoon characters could be semantically classified according to five binary dimensions as American versus European (e.g., Mickey Mouse-Käpt'n Blaubär), as a Disney or non-Disney character (e.g., Donald Duck-Asterix), as a Duck Town resident or living elsewhere (e.g., Donald Duck-Asterix), and as smart or dumb (e.g., Mickey Mouse-Goofy). The names of the characters were classified as consisting of two words or of one (e.g., Hein Blöd-Obelix). 


\section{Procedure and design}

At the beginning of the experiment participants named all cartoon characters shown at a computer screen and subsequently classified each character verbally according to the four semantic categories and according to the name. If necessary, the experimenter provided additional information and feedback. Then a practice block was conducted in which all characters were shown and classified by means of button presses according to each of the five dimensions. The main session with a total of 720 trials consisted of 10 experimental blocks, 2 blocks each for the different classification tasks, being separated by short breaks. Each picture was presented three times per block. At the beginning of each block, the required classification was specified. On each trial, a fixation cross was presented in the middle of the screen for $500 \mathrm{~ms}$, followed by an image presented for $1.8 \mathrm{~s}$; the interstimulus interval was $2.3 \mathrm{~s}$. Participants were instructed to make speeded manual choice responses to each stimulus according to the dimension specified. Responses were accepted if correct and given while the image was presented. Visual feedback was presented after incorrect or missing responses. The images were shown in a randomized sequence. The order of the classification tasks and the classification-to-response mapping was counterbalanced across participants.

\section{Results and discussion}

Mean reaction times (RTs) for correct responses (see Figure 1) and mean error rates in the different classification tasks were submitted to Huyhn-Feldt corrected analyses of variance (ANOVAs) with repeated measurements, performed with both participants $\left(F_{1}\right)$ and stimuli $\left(F_{2}\right)$ as random variables. There were main effects of classification task for correct RTs, $F_{1}(4$, $44)=25.17, M S E=3378.68, p<.001, \varepsilon=.69 ; F_{2}(4,92)=146.69, M S E=1361.51, p<.001$, $\varepsilon=.58$. Post hoc comparisons revealed significant differences between the American versus European $(M=562 \mathrm{~ms})$, Walt Disney $(M=555 \mathrm{~ms})$, and Duck Town classifications $(M=561$ $\mathrm{ms})$ on the one hand and the smart versus dumb $(M=684 \mathrm{~ms})$ and name classification $(M=$ $691 \mathrm{~ms})$ on the other hand $\left(47<F_{1} \mathrm{~s}<57 ; p \mathrm{~s}<.001\right.$, and $\left.180<F_{2} \mathrm{~s}<266\right)$, without any significant differences within these groups of tasks $\left(F_{\mathrm{S}}<1\right)$. Mean error rates were below $10 \%$, and highest in the conditions with longest RTs, excluding an explanation of the RT pattern in terms of speed-accuracy trade-off. ANOVA yielded a main effect of classification task, $F_{1}(4$, $44)=5.57, M S E=8.62, p<.001 ; F_{2}(4,92)=5.62, M S E=24.93, p<.05$. Comparisons between the name classification $(M=8.3 \%)$ and the semantic tasks $(M \mathrm{~s}=5.7,6.7,9.8$, and $4.8 \%$ errors for the American versus European, Walt Disney, smart versus dumb, and Duck Town classification, respectively) failed to reach significance, with the exception of lower error rates for the Duck Town classification $F_{1}(1,11)=6.18, M S E=23.19, p<.05 ; F_{2}(1,23)=$ 11.07, $M S E=25.97, p<.05$.

Experiment 1 was conducted to examine whether adults process names and biographical information about familiar cartoon characters in a similar way as they do in recognizing familiar faces. The general pattern of results replicates earlier findings (Johnston, 1994) in showing strong similarities between familiar faces and cartoon characters. Most important for the current purposes is that adults show the typical advantage of retrieving semantic over name information also when faces of cartoon characters instead of real person's faces are processed. Three of four semantic attributes were classified faster than the names, the fourth (clever vs. dumb) being processed at similar speed. That not all semantic attributes are necessarily classified faster than name attributes had already been observed for a semantic classification of faces (Abdel Rahman et al., 2002). Therefore, this finding is not indicative of a 
difference between cartoon character and face processing. With the expected pattern of reaction times at hand, we now turn to children's performance on semantic and name classification of well-known cartoon characters.

\section{EXPERIMENT 2}

In this Experiment, we examined children with the same tasks and stimuli as those in the previous experiment. If children retrieve semantic and name information in a substantially different way from that of adults, regardless of stimulus familiarity, Scanlan and Johnston's (1997) finding of faster access to names than to semantic information should be replicable also with familiar cartoon characters. In contrast, if the time course of semantic processing and name retrieval in children depends on stimulus or task familiarity rather than developmental changes, the same pattern of retrieval as that in the adults of the present Experiment 1 should be evident.

\section{Method}

\section{Participants}

A total of 12 children ( 9 girls, 3 boys), aged between 8 and 12 years $(M=10)$ were paid for taking part in the experiment. Of these 8 were between 8 and 10 years of age, and 4 were between 11 and 12 years. All children were native German speakers and had normal or corrected-to-normal vision.

\section{Stimuli, procedure, and design}

The stimuli and all other details of the procedure and design were the same as those in Experiment 1. Children's initial performance in the semantic classifications and naming indicated that they were highly familiar with the cartoon characters as well as with the relevant semantic classifications.

\section{Results and discussion}

Mean RTs for correct responses are shown in Figure 1. ANOVA revealed a main effect of classification task, $F_{1}(4,44)=8.3, M S E=4685.97, p<.001, \varepsilon=.85 ; F_{2}(4,92)=33.5, M S E=$ $2163.53, p<.001, \varepsilon=.88$. Post hoc comparisons revealed significant differences between the American versus European $(M=845 \mathrm{~ms})$, Walt Disney $(M=843 \mathrm{~ms})$, and Duck Town classifications $(M=822 \mathrm{~ms})$ on the one hand and the smart versus dumb $(M=924 \mathrm{~ms})$ and name classification $(M=939 \mathrm{~ms})$ on the other hand $\left(9<F_{1} \mathrm{~s}<25\right.$ and $\left.40<F_{2} \mathrm{~s}<76\right)$. Within these groups of tasks, differences were not significant except that the 23- and 22-ms difference between the Duck Town classification and the American/European and Disney classifications, respectively, reached significance in the items analysis. The performance pattern in error rates $(M=8.2,6.8,12.6,6.1$, and $13.3 \%$ for the American vs. European, Walt Disney, smart vs. dumb, Duck Town, and name classification, respectively) mirrored the RT effects without indicating a speed accuracy trade-off. ANOVA revealed a main effect of classification dimension, $F_{1}(4,44)=7.21, M S E=33.57, p<.01, \varepsilon=.55 ; F_{2}(4,92)=12.61, M S E=29.79$, $p<.001, \varepsilon=.715$. Post hoc comparisons revealed the name classification to be more error prone than the Walt Disney and Duck Town classifications, $F_{\mathrm{s}}(1,11)>9$, $p \mathrm{~s}<.05$. 


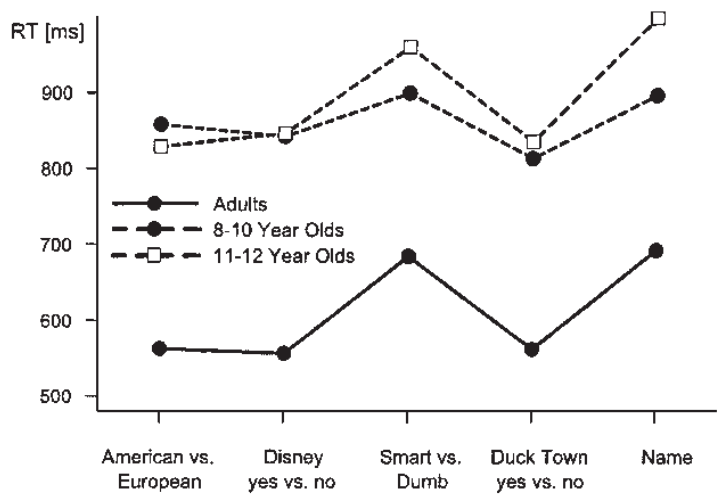

Figure 1. Mean response latencies for the semantic and name classification tasks in Experiment 1 (adults) and Experiment 2 (children).

The present pattern of results in children is remarkably similar to the results of Experiment 1 with adult participants (for a comparison, see Figure 1). In both age groups semantic processing is significantly faster than processing of the characters' names. Only for one semantic classification (smart vs. dumb) did children fail to show an advantage over name classification-but so do adults, as shown in Experiment 1. A repeated measures ANOVA performed with an additional between-subjects factor of age group confirmed the main effect of classification task, $F_{1}(4,88)=28.33, M S E=3621.05, p<.001, \varepsilon=.87 ; F_{2}(4,184)=129.21$, $M S E=1710.46, p<.001, \varepsilon=.79$, and showed that the overall difference in processing speed — with children being slower than adults by $275 \mathrm{~ms}$ on average — is highly significant, $F_{1}(1,22)=43.38, M S E=9630.13, p<.001, F_{2}(1,46)=1547.57, M S E=2642.42, p<.001$. The interaction between task and age group was not significant in the subjects analysis, $F_{1}<1$, but it was in the items analysis, $F_{2}(4,184)=4.42, p<.05$. The latter interaction is most likely caused by the slightly faster reaction times of the children in the Duck Town classification than in the American versus European classification and the Disney classification, which had reached significance in the items analysis of Experiment 1. However, importantly for present purposes, according to the post hoc tests in both age groups the naming task was consistently slower than three of the semantic tasks, and in the fourth semantic task (smart vs. dumb) there was no difference.

Could the results of faster semantic categorization in both age groups be explained by the fact that the Disney, the Duck Town, and the American/European classification split the stimulus set in the same way? All the Disney characters lived in Duck Town and were inevitably American whereas the other alternative held for all the non-Disney characters. Both adults and children might have mapped the stimuli to the corresponding responses without necessarily retrieving the respective types of information. As a result, these classifications would be faster than the name and the smart versus dumb classifications because the latter split the stimuli in a different way, requiring a more controlled nonautomatic mapping of the stimuli to the responses. However, this scenario is unlikely first because the stimulusresponse assignment varied within the experiment. For example, if in the first block of trials Disney characters were classified with a left-hand response and non-Disney characters with a right-hand response, the Duck Town (or American/European) classification in a following 
block had a reversed mapping: Duck Town residents required a right-hand response and so on. Second, a subdivision of the data into whether one of the three fast semantic classifications was required first or was preceded by one or two blocks of a similar classification did not reveal evidence for such an explanation. The Disney classification was even faster when it came first than when it was preceded by either the American $\backslash$ European or the Duck Town classification $\left(M_{\text {diff }}=29 \mathrm{~ms}\right.$ for adults and $112 \mathrm{~ms}$ for children). There was a slight tendency for faster RTs in the opposite direction for the American $\backslash$ European classification $\left(M_{\text {diff }}=-19 \mathrm{~ms}\right.$ for adults but only $-3 \mathrm{~ms}$ for children $)$ and for the Duck Town classification $\left(M_{\text {diff }}=-40 \mathrm{~ms}\right.$ for adults and $135 \mathrm{~ms}$ for children), but all three semantic attributes were classified faster than the names irrespective of the order of these tasks.

We can therefore conclude that children show the same advantage of accessing semantic over name information as do adults when the faces of familiar cartoon characters are processed. These findings suggest that the organization of semantic and name retrieval is not necessarily different in nature between children and adults. Instead, the relative speed of face identification and naming in children appears to depend on the degree of familiarity with the stimulus material or the tasks. If the advantage of name retrieval over semantic processing in children, reported by Scanlan and Johnston (1997), depends on a low degree of familiarity with the stimuli or-alternatively - on a lack of expertise in the semantic tasks, children should show this pattern for faces of (adult's) celebrities. This prediction is directly examined in Experiment 3, which presented faces of politicians who are most likely unfamiliar to children.

\section{EXPERIMENT 3}

This experiment investigated children's identification and naming performance with faces of celebrities of presumably low familiarity to children. If stimulus familiarity is a critical factor in the relative ease of semantic and name classification we should expect a reversed pattern of results as compared to Experiment 2 when politicians instead of cartoon characters are presented. Children should fail to show an advantage of semantic fact over name retrieval —or even show faster name retrieval (cf. Scanlan \& Johnston, 1997).

\section{Method}

\section{Participants}

A total of 12 children ( 7 girls and 5 boys), aged between 8 and 12 years $(M=10)$ were paid for their participation in the experiment. Of these, 7 children were between 8 and 10 years of age, and 5 were between 11 and 12 years. All children were native German speakers and reported normal or correctedto-normal vision.

\section{Stimuli}

Portraits of eight male politicians—-namely, Tony Blair, Benjamin Netanjahu, John Major, Silvio Berlusconi, Oskar Lafontaine, Wolfgang Dressler, Theo Waigel, and Wolfgang Seehofer-were selected as stimuli. All of these were well known in Germany at the time of conducting the experiment (summer 2000). For each politician, three different portraits taken from frontal views were scanned and edited to 8 -bit colour images with a homogeneous grey background. All images were scaled to $3.5 \times 3.5$ 
$\mathrm{cm}$. The politicians could be semantically classified according to their nationality (foreign vs. domestic) and according to their political party membership (government party: Blair, Netanjahu, Lafontaine, and Dressler vs. opposition party: Major, Berlusconi, Seehofer, and Waigel). The surnames of the politicians were classifiable according to the number of syllables (two or less vs. more than two).

\section{Procedure and design}

Because most of the presented politicians were expected to be unknown to the children, the experiment proper was preceded by a learning phase in which all faces were presented on a computer screen together with the names of the politicians, information about their nationality (domestic vs. foreign), and political party membership (government vs. opposition). The children were instructed to study and memorize each face, the associated name and the relevant semantic information. Then the entire set of 24 pictures was presented without additional information, and the children were asked to verbally name and classify each politician according to the previously learned information. This procedure was repeated until all names and relevant semantic attributes were retrieved correctly. In a following practice block all pictures were presented in random order, and the depicted politicians were manually classified according to their nationality, political party membership, and name length. Then, six experimental blocks, separated by short breaks, with a total number of 432 trials were conducted. Each picture was presented three times within each block. At the beginning of each block, the required classification task was specified. On each trial, a fixation cross was presented in the middle of the screen for $500 \mathrm{~ms}$, followed by an image, which was presented for $2.0 \mathrm{~s}$; interstimulus interval was $2.5 \mathrm{~s}$. The children were instructed to make speeded manual choice responses to each stimulus according to the designated dimension. Responses were accepted if correct and given while the image was presented. Visual feedback was presented after incorrect or missing responses. The images were shown in a randomized sequence, and the order of the classification tasks and the classification to response mapping were counterbalanced across participants.

\section{Results and discussion}

Mean RTs for correct responses in the different classification tasks are shown in Figure 2. A Huyhn-Feldt corrected repeated measures ANOVA with the three experimental conditions yielded a main effect of classification task for correct RTs, $F_{1}(2,22)=5.35, M S E=2364.55$, $p<.01, \varepsilon=.97 ; F_{2}(2,46)=14.11, p<.001, M S E=1596.72, \varepsilon=.95$. Post hoc comparisons revealed that names were classified significantly faster than political party membership $(M=$ 971 vs. $1026 \mathrm{~ms}), F_{1}(1,11)=5.54, M S E=6614.74, p<.05 ; F_{2}(1,23)=19.85, M S E=3550.4$, $p<.001$, whereas the name and nationality classifications $(M=971 \mathrm{~ms}) \operatorname{did} \operatorname{not} \operatorname{differ}\left(F_{\mathrm{S}}<1\right)$. An ANOVA conducted on mean error rates $(M=10.3,14.8$, and $13.4 \%$ for the foreign vs. domestic, government vs. opposition, and name classification, respectively) yielded no main effect of classification task $\left(F_{\mathrm{S}}<1\right)$.

This experiment investigated whether children show a similar advantage of semantic over name classifications as that shown in Experiment 2 when relatively unfamiliar faces of politicians are presented. Clearly, this is not what we observed. Instead, the results replicate the finding of Scanlan and Johnston (1997) in showing that children may retrieve names faster than semantic information. For the specific classification tasks employed in this experiment, naming latencies were either as fast as the semantic classification (nationality) or faster (political party membership). 
A direct comparison with the performance of adult participants in the same task as that of the children in the present Experiment 3 is available from Abdel Rahman et al. (2002; see Figure 2, bottom), which illustrates the difference between the children and adults. Whereas adults produced the typical pattern of faster semantic than name classifications, children showed the opposite effect. This impression is confirmed by an additional ANOVA including the within-subjects factor of classification task (nationality, political party membership, and name) and a between-subjects factor of age group. There were main effects of classification task, $F_{1}(2,44)=15.65, M S E=2272.97, p<.001, \varepsilon=1.0 ; F_{2}(2,92)=73.45, M S E=899.4, p<$ .001 , and age group, $F_{1}(1,22)=92.56, M S E=21,024.88, p<.001 ; F_{2}(1,46)=1251.1, M S E=$ $3204.21, p<.001$, and-most important-a strong interaction between these variables, $F_{1}(2$, $44)=8.9, p<.001 ; F_{2}(2,92)=47.85, p<.001$, which reflects the reversal of the naming advantage in children to a disadvantage in adults.

Taken together, the current experiments demonstrate that children show a naming advantage only when presented with unfamiliar faces but not when stimuli and semantic dimensions are familiar. When retrieving semantic information about familiar stimuli they show exactly the same advantage over name information as that shown by adults. These findings suggest that there is no principal difference in the cognitive architecture of children and adults. Following this interpretation the question arises of whether adults would show a similar naming advantage as that shown by children when confronted with newly learned faces. This appears to be rather unlikely because Cohen (1990) and McWeeny, Young, Hay, and Ellis (1987) reported the typical naming disadvantage for adults also in learning experiments where it took longer to learn the name of a face than associated biographical information. This held true even when the same words designated semantic attribute and name; for example "baker" was learned slower when designating a name than when designating a profession (McWeeny et al., 1987). Adults should therefore retrieve semantic information such as nationality or profession faster than names even when the information has been newly acquired.

Now, given our claim that there is no fundamental difference between adults and children, as suggested by the present experiments and, furthermore, given that adults are unlikely to show a naming advantage when presented with newly learned faces, what is the critical determinant for the time course of semantic and name retrieval in both age groups? As suggested earlier it might be the degree of expertise in the relevant semantic domain - in other words, the familiarity with the semantic concept - rather than the novelty of the information per se that causes the semantic disadvantage observed in children. This explanation will be explored in the final experiment in which adults are confronted with new faces for which names and rather uncommon semantic information were presented.

\section{EXPERIMENT 4}

Here, we tested the expertise account of the relative time course of semantic and name retrieval. If a lack of expertise with the relevant semantic domains causes the retrieval disadvantage rather than stimulus familiarity per se, we should observe faster naming in adults when little-known semantic information is presented. In order to equate the experimental situation of the adults to the situation of the children in Experiment 3, we presented faces of unfamiliar persons in a learning experiment in which names and uncommon semantic information about nationality and occupation was given. 


\section{Method}

\section{Participants}

Seven females and four males, aged 19 to 31 years $(M=26)$ were paid for their participation in the experiment or received partial fulfilment of a curriculum requirement. All participants were native German speakers and reported normal or corrected-to-normal vision. Three participants with error rates of more than $30 \%$ had to be replaced.

\section{Stimuli}

Portraits of eight unfamiliar male persons were selected as stimuli. For each person, three different portraits taken from frontal views were edited to 8-bit colour images with a homogeneous grey background. All images were scaled to $3.5 \times 3.5 \mathrm{~cm}$. The persons were in equal numbers assigned to the two semantic dimensions of nationality and occupation. For the nationality classification we chose the islands of Aruba and Vanuatu, both little known to German participants; as occupations we chose Hafner (stovefitter) and Sphragist (sigillograph) because we expected that very little is known about these rare but existent professions. For each face a name was selected that could be classified according to the number of syllables (two or less vs. more than two); the names were Mastarrigo, Hilsamer, Koley, Boge, Ruscheinski, Monnerjarn, Neufs, and Orth. The three dimensions were assigned in an orthogonal fashion across the faces.

All other details of the procedure and design were identical to those in Experiment 3.

\section{Results and discussion}

Mean RTs for correct responses in the different classification tasks are shown in Figure 2, right panel. A Huyhn-Feldt corrected repeated measures ANOVA with the three experimental conditions yielded a main effect of classification task for correct RTs, $F_{1}(2,22)=3.4$, $M S E=3948.07, p<.05, \varepsilon=1 ; F_{2}(2,46)=22.88, p<.001, M S E=1153.87, \varepsilon=.76$. Post hoc comparisons revealed faster classification latencies for the names than for the occupations $(M \mathrm{~s}$ $=717 \mathrm{~ms}$ vs. $759 \mathrm{~ms}), F_{1}(1,11)=2.6, M S E=7967.03, p<.10 ; F_{2}(1,23)=11.7, M S E=$ $3521.08, p<.01$. The name and nationality classifications $(M=693 \mathrm{~ms})$ did not differ in the subjects analysis, $F_{1}(1,11)<1$, but did so in the items analysis, $F_{2}(1,23)=6.0, M S E=$ $2314.97, p<.05$.

An ANOVA conducted on mean error rates $(M \mathrm{~s}=4.1,7.6$, and $4.8 \%$ for the nationality, occupation and name classifications, respectively) yielded a similar pattern with a main effect of classification task, $F_{1}(2,22)=3.3, M S E=12.2, p<.05, \varepsilon=1 ; F_{2}(2,46)=4.8, p<.05, M S E$ $=23.09, \varepsilon=.73$. There was a slight trend for higher error rates in the occupation classifications than in both the nationality and the name classifications which did not reach conventional levels of significance. Because the error rates tended to be higher in the conditions with slower RTs a speed-accuracy trade-off cannot explain the differences in reaction times between conditions.

The results of this experiment show that not only children but also adults retrieve names faster than semantic information if they have very little knowledge concerning the semantic information to be retrieved. After the experiment the participants were asked whether they knew where Aruba and Vanuatu were located and whether they knew anything about the occupations presented. Only two participants knew Aruba and Vanuatu, and none of them was familiar with the occupations associated with the unknown faces. Thus, even when the 

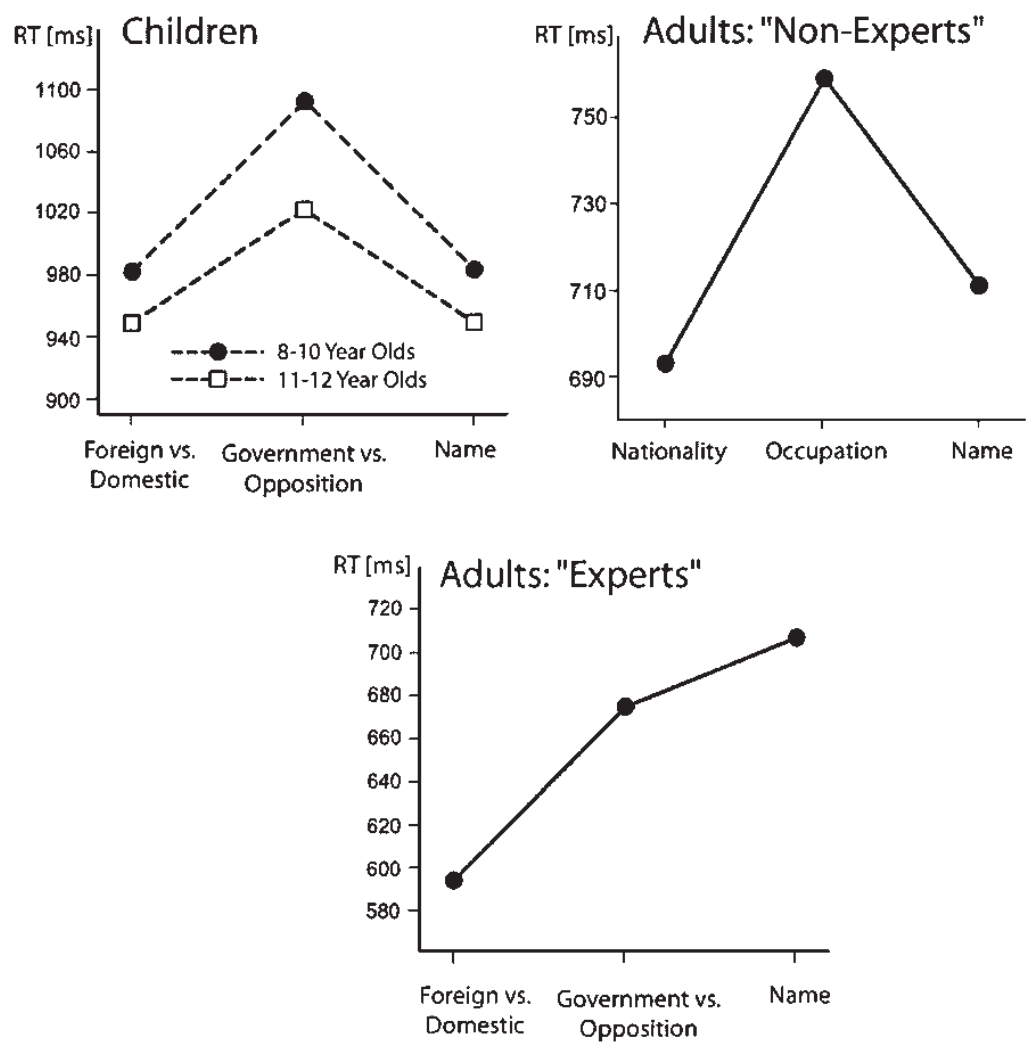

Figure 2. Top: mean response latencies for the semantic and name classification tasks in Experiment 3 for children (left panel) and Experiment 4 for adults (right panel). Bottom: data from Abdel Rahman, Sommer, and Schweinberger (2002), for adult "experts".

semantic categories (i.e., nationality and occupation) are very basic and concrete, a lack of expertise regarding the specific information can turn the typically observed semantic advantage over names into a disadvantage. This result is all the more surprising because there were only two different nationalities and occupations presented in contrast to eight different (and rather uncommon) names. Thus, the relative time course of semantic and name retrieval is strongly influenced by the degree of expertise with the specific semantic concept.

\section{GENERAL DISCUSSION}

The goal of this study was to reassess an important piece of evidence against the serial arrangement of access to semantic facts and names of faces. Scanlan and Johnston (1997) reported that children retrieved names faster than semantic information about familiar faces, which is at variance with the naming disadvantage commonly found in adults. In the present study we tested whether the naming advantage of children holds true also for more familiar stimuli and tasks. When using cartoon characters, both children and adults showed the common naming disadvantage for three of four semantic tasks, indicating that for familiar stimuli there is no 
difference in the cognitive architecture in children and adults. Therefore it appears that familiarity or expertise with a semantic concept is an important determinant in children's performance. That this is the case was shown in Experiment 3, which replicated the naming advantage of children relative to the retrieval times of rather unfamiliar semantic information associated with newly learned faces (political party membership). Together, these findings suggest that child and adult naming performance is very similar when the stimulus material or the semantic classification tasks are familiar. Only when this is not the case do the striking performance differences between children and adults, reported by Scanlan and Johnston, appear.

Therefore, the findings of Scanlan and Johnston (1997) and Experiment 3 in the current study do not indicate a genuine difference in face processing between children and adults for relatively unfamiliar material or tasks. Interestingly, although the name classification in Experiment 3 was relatively complex (syllable classification) the classification of the semantic dimensions (nationality and political party membership) appeared even more demanding for children resulting in longer retrieval times. Thus, the complexity of the semantic attributes appears to strongly influence children's performance on these tasks. This suggestion is supported by a between-group comparison of the children's reaction times for cartoon characters (Experiment 2) and politicians (Experiment 3). On average, name decision latencies for the cartoons were somewhat faster than those for politicians by $32 \mathrm{~ms}$; a statistical test indicated this difference to be significant in the items but not in the subjects analysis, $t_{1}(46)=3.52, p<.001$, and $t_{2}(22)<1$, respectively. Therefore, in line with the suggestion put forward by Scanlan and Johnston, the naming advantage found by the authors and in the present Experiment 3 can be attributed to the children's problems with the semantic tasks rather than to any special advantage for name processing. Because comparable difficulties with semantic dimensions were not apparent for children in Experiment 2, the most plausible assumption is that it was the lack of expertise with the semantic dimensions or concepts tested by Scanlan and Johnston and present Experiment 3 that posed the problems. For example, children at the age of our participants may not have a vivid representation for political party membership; they do, however, know quite well what a Disney cartoon is. Furthermore, they most probably know several cartoon characters sharing the same semantic attribute, while their knowledge about politicians might be confined to the information acquired in the experiment.

Experiment 4 further explored the semantic expertise account of the observed differences between adults and children. Because several studies with adults have demonstrated the typical semantic retrieval time advantage also for previously unfamiliar faces in learning experiments (Cohen, 1990; McWeeny et al., 1987) it is unlikely that stimulus familiarity per se is the determining factor. Therefore, we presented unfamiliar face stimuli in a learning experiment together with rather uncommon and infrequent semantic information. In this situation adults showed a similar RT pattern as that shown by children when confronted with unfamiliar face stimuli (Experiment 3) -namely, faster retrieval of names than of semantic attributes. This result is particularly surprising because, even though the semantic features were not well known, they belonged to familiar semantic dimensions (nationality and occupation) that are typically retrieved faster than proper names.

Together, the results of four experiments suggest that in both children and adults the relative ease of semantic information and name retrieval depends on the level of expertise with the 
specific types of information rather than age differences or stimulus familiarity per se. These findings are easy to accommodate with modern IAC models of face recognition, assuming that semantic information is usually retrieved faster than names because of the superior connectivity for many kinds of semantic information. Within this framework, it is easy to conceive that for some kinds of semantic information the connections might be as weak as or even weaker than those for names, yielding slower reaction times and higher error rates. This may be the case for some of the semantic classification dimensions required from the children in the present Experiment 3 and in the study of Scanlan and Johnston, and for adults in Experiment 4 .

The pattern of expertise-dependent inferior or superior name retrieval times provides converging evidence to the findings reported by Scanlan and Johnston (1997) and Abdel Rahman et al. (2002) against the common notion of a general naming advantage over semantic fact retrieval. Serial processing models might account for these findings by suggesting that only specific types of semantic information have to be accessed as a necessary prerequisite for name retrieval. Given this assumption one might argue that the specific pieces of semantic information retrieved in Experiments 3 with children and in Experiment 4 with adults (political party membership and occupation) do not belong to the class of minimal necessary and sufficient semantic information for naming. Whereas it is very plausible that not all the available semantic information about a person is activated by his or her face, it is hard to derive explicit predictions about which types of semantic information mediate naming and which types of information can be retrieved in parallel.

A diary study on everyday difficulties in person identification and naming (Young et al., 1985) has shown that in situations in which only partial information was retrieved, knowledge about the person's occupation and where he or she was usually seen was most commonly spared. The observation that persons can be named in the context of partial semantic information (e.g., a person is identified and named as an actor without information about the particular movies the person acted in) strongly suggests that not all semantic information is necessary for naming. Importantly, however, there was no instance of successful name retrieval in the absence of information about occupation or place where the person is usually seen. Thus, occupation and place might be promising candidates for the minimal and necessary semantic information accessed in naming.

Similarly, neuropsychological evidence about which semantic information might be crucial for name retrieval suggests that occupation could be such a critical feature. In a study with 24 patients with dementia of Alzheimer type, Hodges and Greene (1998) describe instances of naming in the context of minimal semantic information. The preserved semantic information concerned occupation more or less directly in almost every case (e.g., "politician", "Prime Minister", "in films", "boss of Egypt", or "dictator abroad").

Under the assumption that occupation is a critical piece of information mediating name retrieval the finding of faster naming compared to the retrieval of (low frequent) occupation information in Experiment 4 is difficult to reconcile with the notion of serial processing. It would require additional assumptions, such as, for example, context-dependent semantic mediation - for example, whatever minimal piece of semantic information available mediates name retrieval. In this case the experimental test of serial processing becomes an increasingly difficult endeavour. Consequently, our findings are not inherently incompatible with serial processing but they do cast doubt on the notion of predefined and context-independent 
semantic features mediating proper name retrieval. They are, however, easily accounted for within the context of modern IAC models, which postulate a parallel activation of units representing semantic and name information.

In conclusion, we have shown that although there appears to be no principal difference in face processing between children and adults, identification and naming performance is strongly affected by the level of expertise with the semantic tasks or concepts called for. When children are familiar with the relevant semantic concepts, their performance pattern is very similar to that of "expert" adults_ apart from a general slowing. Similarly, when adults are required to retrieve semantic information for which they do not have a vivid representation, their performance resembles the performance of "non-expert" children.

\section{REFERENCES}

Abdel Rahman, R., Sommer, W., \& Schweinberger, S. R. (2002). Brain-potential evidence for the time course of access to biographical facts and names of familiar persons. Fournal of Experimental Psychology: Learning, Memory, and Cognition, 28, 366-373

Brédart, S. (1993). Retrieval failures in face naming. Memory, 1, 351-366.

Brédart, S., Valentine, T., Calder, A., \& Gassi, L. (1995). An interactive activation model of face naming. Quarterly Journal of Experimental Psychology, 48A, 466-486.

Brennen, T., David, D., Fluchaire, I., \& Pellat, J. (1996). Naming faces and objects without comprehension: A case study. Cognitive Neuropsychology, 13, 93-110.

Bruce, V., \& Young, A. (1986). Understanding face recognition. British fournal of Psychology, 77, 305-327.

Burton, A. M., \& Bruce, V. (1992). I recognize your face but I can't remember your name: A simple explanation? British Fournal of Psychology, 83, 45-60.

Burton, A. M., \& Bruce, V. (1993). Naming faces and naming names: Exploring an interactive activation and competition model of person recognition. Memory, 1, 457-480.

Burton, A. M., Bruce, V., \& Hancock, P. J. B. (1999). From pixels to people: A model of familiar face recognition. Cognitive Science, 23(1), 1-31.

Cohen, G. (1990). Why is it difficult to put names to faces? British fournal of Psychology, 81, 287-297.

Cycowicz, Y. M., Friedman, D., Rothstein, M., \& Snodgrass, J. G. (1997). Picture naming by young children: Norms for name agreement, familiarity, and visual complexity. Fournal of Experimental Child Psychology, 65, 171-237.

Fery, P., Vincent, E., \& Brédart, S. (1995). Personal name anomia: A single case study. Cortex, 31, 191-198.

Graf, P. (1990). Life-span changes in implicit and explicit memory. Bulletin of the Psychonomic Society, 28, 352-358.

Hanley, J. R. (1995). Are names difficult to recall because they are unique? A case study of a patient with anomia. Quarterly Journal of Experimental Psychology, 48A, 487-506.

Hanley, J. R., \& Cowell, E. S. (1988). The effects of different types of retrieval cues on the recall of names of famous faces. Memory E Cognition, 16, 545-555.

Hodges, J. R., \& Greene, J. D. W. (1998). Knowing about people and naming them: Can Alzheimer's disease patients do one without the other? Quarterly Fournal of Experimental Psychology, 51 A, 121-134.

Johnston, R. A. (1994). Some Mickey Mouse experiments: An examination of the classification and naming of individual things. British fournal of Psychology, 85, 525-540.

Johnston, R. A., \& Bruce, V. (1990). Lost properties? Retrieval differences between name codes and semantic codes for familiar people. Psychological Research/Psychologische Forschung, 52, 62-67.

Luchelli, F., \& De Renzi, E. (1992). Proper name anomia. Cortex, 28, 221-230.

McClelland, J. L., \& Rumelhart, D. E. (1981). An interactive activation model of context effects in letter perception: Part 1. An account of basic findings. Psychological Reviem, 88, 375-407.

McWeeny, K. H., Young, A. W., Hay, D. C., \& Ellis, A. W. (1987). Putting names to faces. British Fournal of Psychology, 78, 143-149.

Scanlan, L. C., \& Johnston, R. A. (1997). I recognize your face, but I can't remember your name: A grown-up explanation? Quarterly Fournal of Experimental Psychology, 50A, 183-198. 


\section{ABDEL RAHMAN, SOMMER, OLADA}

Schweinberger, S. R., Burton, A. M., \& Kelly, S. W. (2001). Priming the access to names of famous faces. British Fournal of Psychology, 92, 303-317.

Semenza, C., \& Zettin, M. (1988). Generating proper names: A case of selective inability. Cognitive Neuropsychology, $5(6), 711-721$.

Semenza, C., \& Zettin, M. (1989). Evidence from aphasia for the role of proper names as pure referring expressions. Nature, 342, 678-679.

Valentine, T., Brennen, T., \& Brédart, S. (1996). The cognitive psychology of proper names. London, UK: Routledge.

Valentine, T., Hollis, J., \& Moore, V. (1998). On the relationship between reading, listening, and speaking: It's different for people's names. Memory E Cognition, 26, 740-753.

Young, A., Ellis, A. W., \& Flude, B. M. (1988). Accessing stored information about familiar people. Psychological Research, 50, 111-115.

Young, A. W., Hay, D. C., \& Ellis, A. W. (1985). The faces that launched a thousand slips: Everyday difficulties in recognizing people. British Journal of Psychology, 76, 495-523.

Young, A. W., McWeeny, K. H., Ellis, A. W., \& Hay, D. C. (1986). Naming and categorizing faces and written names. Quarterly Journal of Experimental Psychology, 38A, 297-318.

Original manuscript received 12 November 2002

Accepted revision received 23 April 2003

PrEviem proof published online 4 September 2003 